\title{
Mobility Conscious Medium Access Control Scheme for Wireless Sensor Networks: A Conceptual Approach
}

\author{
Adeniran Ishola Oluwaranti ${ }^{1}$, Tayo Dorcas Obasanya ${ }^{2}$ \\ ${ }^{1}$ Department of Computer Science and Engineering, Obafemi Awolowo University, Ile-Ife, Nigeria \\ ${ }^{2}$ Department of Computer Engineering, Ekiti State University, Ado-Ekiti, Nigeria \\ Email: nirano@gmail.com, tjoyfdt@yahoo.com
}

Received 16 May 2014; revised 16 June 2014; accepted 15 July 2014

Copyright (C) 2014 by authors and Scientific Research Publishing Inc.

This work is licensed under the Creative Commons Attribution International License (CC BY). http://creativecommons.org/licenses/by/4.0/

c) (i) Open Access

\begin{abstract}
Mobility in Wireless Sensor Network (WSN) presents distinctive challenges in Medium Access Control (MAC) scheme. Numerous MAC protocols for sensor networks assume that sensor nodes are static and focus primarily on energy efficiency. This work seeks to develop an improved mobility conscious medium access control scheme for wireless sensor networks with a view to enhance energy conservation on mobile sensor nodes. On this note, mobility patterns of different scenarios are modelled using Gauss Markov Mobility Model (GMMM) to determine the position and distance of the sensor nodes and how they are correlated in time.
\end{abstract}

\section{Keywords}

Mobility, Medium Access Control, Wireless Sensor Networks, Gauss Markov Mobility Model

\section{Introduction}

Recent technological advancements have made the deployment of small, inexpensive, low-power devices a reality. Wireless Sensor Networks (WSN) has emerged as one of the first real applications of ubiquitous computing. It plays a key role in bridging the gap between the physical and the computational world by providing reliable, scalable, fault tolerant, and accurate monitoring of physical and environmental conditions [1]. The development of WSN was originally motivated by military applications such as battlefield surveillance. In recent times, they are used in many applications ranging from health such as tracking and monitoring doctors and patients to environmental such as precision agriculture, home application such as smart environment, commercial application such as detecting and monitoring car thefts [2]. Furthermore, WSN is a wireless network consisting of spatially 
distributed sensor nodes to cooperatively monitor a region to obtain data about the environment, physical or environmental conditions such as temperature, sound, vibration, pressure, motion or pollutants at different locations.

Figure 1 shows a typical deployment of sensor nodes in data gathering applications. Sensors are typically scattered in a field. The scattered sensor nodes are deployed randomly in a sensor field. Sensor nodes collect and route data, which is aggregated back to the Sink by multi-hops. In this figure, node A communicates with Sink to deliver messages, it will route data from A to E or from E to A by multi-hops. The Sink communicates with the task manager node through Internet or satellite link to deliver data that is aggregated. The task manager node also uses the collected data to make relevant analysis and applications. A user makes use of any display interface (i.e. PDA, computers) to retrieve or analyze these information locally or remotely.

In WSN, to extend the battery lifetime, protocols at the data link layer usually put sensors in a sleep mode most of the time, and only let nodes wake up periodically for data communication. This mode of operation, which trades network metrics for energy saving, proves to be very effective in stationary network, where connection formations and break-ups are rare events. However, these protocols, which are customized for stationary networks, do not work well in mobile scenarios such as a patient assistance system which monitors patients' health conditions via wearable bio-sensors, workers equipped with sensor device in disaster recovery situation, soldiers in battle field, and to provide these with appropriate care when needed [3].

In most of the existing works, efforts have been made to develop MAC protocols that primarily conserve energy assuming static nodes. They failed to consider real life situations where some nodes are mobile hence this renders the existing schemes inefficient when it comes to mobility. However, for the purpose of this work Gauss Markov mobility model is adopted. This work will also use Clear Channel Assessment (CCA) and packets back-off for channel negotiation and Low Power Listening (LPL) for low power communication and also mobility patterns for node awareness. This is with a view to check the status of the channel and report back if there is activity. The rest of this paper is organized as follows. In Section 2, we presented reviews of related works. We described the overview of a Mobility Conscious Medium Access Control (MAC) Scheme in Section 3. In Section 4, the model formulation is presented while Section 5 offers concluding thoughts and future work.

\section{Related Works}

A MAC protocol is required in sensor networks to coordinate the sensor nodes' access to the shared medium [4]. Many works have been and are being done on medium access control (MAC) protocols for wireless sensor networks.

The work done by [5] on Sensor Medium Access Control (S-MAC), a Medium Access Control (MAC) protocol was specifically designed for WSN. The main objective of S-MAC is to conserve energy in sensor networks. It takes into consideration that fairness and latency are less critical issues compared to energy savings. The basic idea of S-MAC is that time is divided into frames. It uses a listen/sleep cycle to reduce energy consumption. It forces sensor nodes to operate at low duty cycle by putting nodes into periodic sleep instead of idle listening. Although, SMAC saves more power than 802.11, it does not adapt to network traffic very well since it uses a fixed duty cycle for all the sensor nodes.

Timeout MAC (T-MAC) proposed by [6] is a contention based MAC protocol. T-MAC improves on S-MAC

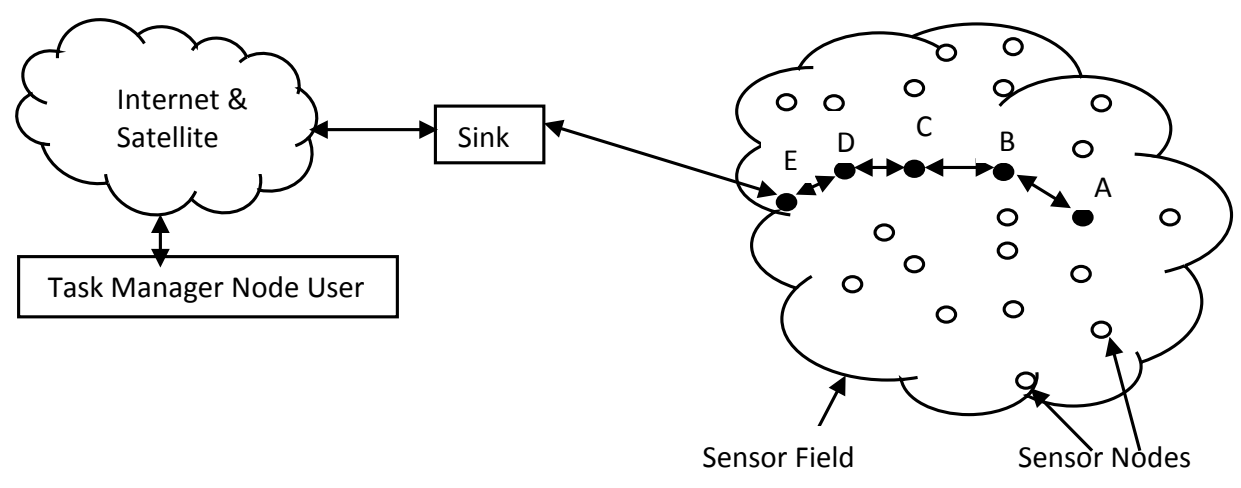

Figure 1. Typical WSN deployment in data gathering applications, [2]. 
by using an adaptive duty cycle. This means that if there is no activity in the vicinity of a node for a threshold time, the node goes to sleep. Such an adaptation frees the application from the burden of selecting an appropriate duty cycle. TMAC has the same performance as SMAC under constant traffic loads, but saves more energy under variable traffic. The downside of TMAC's aggressive power conserving policy is that nodes can go to sleep rather early, resulting in increased latency and lower throughput. Another drawback in both SMAC and TMAC is they group the communication during small periods of activity. As a result, the protocols collapse under high traffic loads.

Traffic Adaptive MAC (TRAMAC) was developed by [7] for energy efficient and collision free channel access in wireless sensor networks. It uses the Time Division Multiple Access (TDMA) scheme by dividing time into slots. It assumes that time is slotted and uses a distributed election scheme based on information about the traffic at each node to determine which node can transmit at a particular time slot. It uses traffic-based information to decide on schedules for individual nodes and thus is adaptive to network traffic.

WiseMAC was proposed by [8] and uses a preamble sampling technique called Low Power Listening (LPL), where a node regularly samples or polls the medium for a very brief time to check whether a packet needs to be received. When the node wakes up and senses no traffic, it again goes to sleep mode. If the source has to send some data, it transmits a wakeup preamble. If a node polls the medium and senses the wakeup preamble, it receives the data and acknowledges and again goes to sleep mode. The decentralized sleep-listen scheduling in WiseMAC results in different sleep and wakeup times for each neighbour of a node. This is especially an important problem for broadcast type of communication, since broadcasted packet will be buffered for neighbours in sleep mode and delivered many times as each neighbour wakes up. However, this redundant transmission will result in higher latency and power consumption.

Berkely MAC (B-MAC) was introduced by [9]. B-MAC is a carrier sense media access protocol that uses an adaptive preamble sampling scheme to reduce duty cycle and minimize idle listening. B-MAC uses a technique call Low Listening Power (LLP) to reduce energy consumption. Nodes are awakening at fixed interval (check interval) to check the wireless channel for valid preamble bytes that indicate a pending data transmission from another node.

The limitation is during connection setup time, the mobile node is disconnected from the rest of the network, and thus these mobile nodes may not create a new connection fast enough and will degrade network performance. Thus limited energy resource of sensor nodes will not be efficiently manage, because B-MAC does not take into consideration, mobility of some of the sensor nodes.

The work of [10] was on Lightweight MAC (LMAC). Lightweight medium access protocol uses a TDMA scheme, which provides collision-free communication. It is less energy efficient because each node has to listen for control messages during the whole frame. Besides simplicity, an important design goal was to minimize the number of transitions between receive and transmit mode, because they take time during which the radio cannot be used. Only one time slot is assigned to each node and the node is given control over the time slot. This increases the latency because each node has to wait for its time slot. If there is a new node joining the network it will listen to the whole frame before sending the data. It uses the control packet to inform the other nodes about the route, current time slot etc.

Traffic Aware Energy Efficiency MAC (TEEM) which was also inspired from S-MAC was developed by [11]. In order to reduce the energy consumption, in this work, nodes are not active during the complete sleep period. It goes to sleep directly when no data packets are buffered to be transmitted. TEEM also has more packet collision than S-MAC and TMAC, this is the reason for end to end delay realized which increases exponentially when the node density increases.

Pattern MAC (PMAC) was proposed by [12]. According to the design concept of PMAC the sensor generate an appropriate sleeping schedule based on its own data transfer condition. The objective of these works is mainly to efficiently conserve energy while trading off parameters like latency, fairness and throughput. Meanwhile it obtains the sleep/wake schedule of the neighbouring sensors by the Pattern Exchange process with the neighbouring sensors. PMAC when compared to S-MAC produces higher data throughput and saves more energy.

Mobile Lightweight MAC (MLMAC) proposed by [13] is a modification of the work done by [10] LMAC. It is a TDMA based MAC protocol for mobile wireless sensor networks where all sensor nodes are mobile. The main difference between LMAC and MLMAC is their intended scenario. While LMAC assumes a static sensor network where communication takes place between nodes and the gateway, MLMAC scenario display that all nodes are mobile and communicate among each other. MLMAC does not depend on a gateway to start the syn- 
chronization; instead, it is fully dynamic.

\section{Overview of a Mobility Conscious Medium Access Control (MAC) Scheme}

Based on the work of [9], there are limitations when the sensor nodes are mobile. BMAC allows each node to wake up occasionally to check for channel activity. The wake-up period is referred to as the check interval. The check interval used by B-MAC assumes nodes are static. During connection setup time, the mobile node is disconnected from the rest of the network, thus these mobile nodes may not create a new connection fast enough. Thus limited energy resource of sensor nodes will not be efficiently manage, because B-MAC does not take into consideration, mobility of some of the sensor nodes. This paper is proposing a mobility conscious scheme to enhance the work done by [9].

In wireless communication networks, medium access control schemes are used to manage the access of active nodes in a shared channel. Energy and throughput of MAC protocols may significantly affect the overall performance of a wireless sensor network.

\subsection{Mobility in Wireless Sensor Networks}

Mobility in sensor networks brings the following issues in the design of protocols in different layers:

1) In a mobile network, the position of sensor nodes changes over time. A mobility conscious protocol needs to estimate the position of each node in the network at any point in time.

2) The movement of nodes can cause frame errors in the network. A mobile protocol needs to handle these frame errors and retransmit frames where necessary.

3) In the case of routing protocols, node mobility can result in route changes, which will lead to delivery failures if not propagated in time. To lessen the problem, mobility information needs to be used in route calculation. To this end, neighbours table and route maintenance should adapt to the mobility in networks.

4) In the case of schedule based MAC protocols, a neighbourhood inconsistency of two-hop neighbours information can occur when mobile nodes enter or leave the neighbourhood. This leads to schedule inconsistencies [14]. A mobility conscious MAC protocol needs to utilize the mobility information of nodes and determines which nodes enter or leave the neighbourhood to eliminate these inconsistencies.

\subsection{Mobility Patterns}

Mobility pattern is the actual set of movements that result from applying the mobility model to one, or more nodes. It plays a significant role in determining protocol performance therefore, it is necessary for mobility models to emulate the movement pattern of targeted real life applications in a reasonable way. Similarly, a mobility pattern is the movement pattern of real life objects such as people or vehicles. According to [15], a mobility pattern is described by properties like dimension, limitation, group behaviour and predictability.

\section{Description of the Proposed Model}

The proposed work uses Clear Channel Assessment (CCA) and packets back-off for channel negotiation, and Low Power Listening (LPL) for Low Power Communication (LPC) and also Mobility patterns for node awareness. In order for nodes to be aware of mobile nodes, there is need for a mobility model. The model used in this research work represents a wide range of user mobility patterns including linear motion, random motion and totally random motion. For the purpose of this work Gauss Markov mobility model is used. The proposed model considers both static and mobile nodes and generates mobility patterns for mobile nodes to be integrated as part of the network. It then uses a threshold distance to change the check interval of the channel so as to provide ease of communication. This is an improvement over B-MAC. It will minimize the energy consumed when communicating with other nodes.

\section{Proposed Mobility Model}

The Gauss-Markov Mobility Model was first introduced by [16] for the simulation of a Personal Communication Service (PCS) network and was subsequently used for the simulation of an ad hoc network protocol by [17]. The model has since been widely utilized. Gauss-Markov mobility model was used for the work because the 
model is capable of duplicating different kinds of mobility behaviour in various scenarios and models. It does this using a tuning parameter to vary the degree of randomness. The proposed model considers both static and mobile nodes and generates mobility patterns for mobile nodes to be integrated as part of the network. It then uses a threshold distance to change the check interval of the channel so as to provide ease of communication. Also the next movement of the mobile nodes is dependent on its previous movement. That is the movement of the mobile nodes is correlated in time. The model was designed with a tuning parameter $(\alpha)$. The tuning parameter affects the randomness of the behaviour of a mobile node. In this model, a sensor node's velocity is assumed to be correlated in time and modeled by a Gauss-Markov random process. In continuous time, a stationary Gaussian process $v(t)$ is a Gauss-Markov process if it has the autocorrelation function. This is as shown below:

$$
R_{v}(\tau)=\boldsymbol{E}[v(t) v(t+\tau)]=\sigma^{2} \mathfrak{e}^{-\beta|\tau|}+\mu^{2}
$$

where $\sigma^{2}$ is the variance of $v(t), \mu$ is its mean and $\beta \geq 0$ determines the degree of memory.

The discrete version of the mobile velocity is defined with $v_{n}=v(n \Delta t)$, and the memory level $\alpha=\mathrm{e}^{-\beta \Delta t}$, where $\Delta t$ is normalized to unity. The discrete Gauss-Markov process in equation (i) can be represented by the following recursive realization:

$$
V_{n}=\alpha V_{n-1}+(1-\alpha) \mu+\sigma \sqrt{1-\alpha^{2}} W_{n-1}
$$

where $\left\{w_{n}\right\}$ is an uncorrelated Gaussian process with zero mean and unit variance and is independent of $\left\{v_{n}\right\}$. Initially each mobile node is assigned an initial speed and direction. At fixed intervals of time, $n$, movement occurs by updating the speed and direction of each mobile node. Specifically, the value of speed and direction at the $n^{\text {th }}$ instance is calculated based on the value of speed and direction at the $(n-1)^{\text {th }}$ instance i.e. the previous instance and a random variable using the following equations according to [18]:

$$
\begin{aligned}
& s_{n}=\alpha s_{n-1}+(1-\alpha) \bar{s}+\sqrt{1-\alpha^{2}} s_{x n-1} \\
& d_{n}=\alpha d_{n-1}+(1-\alpha) \bar{d}+\sqrt{1-\alpha^{2}} d_{x n-1}
\end{aligned}
$$

where $s_{n}$ and $d_{n}$ are the new speed and direction of the mobile node at time interval $n ; \alpha$, where $0 \leq \alpha \leq 1$, is the tuning parameter used to vary the randomness; $\bar{s}$ and $\bar{d}$ are constants representing the mean value of speed and direction as $n \rightarrow \infty$; and $s_{x n-1}$ and $d_{x n-1}$ are random variables from a Gaussian distribution with mean equal to zero and standard deviation equal to one.

At each time interval the next location is calculated based on the current location, speed and direction of movement. Specifically, at time interval $n$, a mobile node's position is given by the equations:

$$
\begin{aligned}
& x_{n}=x_{n-1}+s_{n-1} \cos d_{n-1} \\
& y_{n}=y_{n-1}+s_{n-1} \sin d_{n-1}
\end{aligned}
$$

where $\left(x_{n}, y_{n}\right)$ and $\left(x_{n-1}, y_{n-1}\right)$ are the $x$ and $y$ coordinates of the mobile node's position at the $n^{\text {th }}$ and $(n-1)^{\text {th }}$ time intervals, respectively, and $s_{n-1}$ and $d_{n-1}$ are the speed and direction of the mobile node respectively at the $(n-1)^{\text {th }}$ time interval [19].

In this work, the nodes position in $x$ and $y$ dimension will be calculated as well as the distance dist, between each nodes. This is given by:

$$
\text { dist }=\sqrt{\left(x_{n}-x_{n-1}\right)^{2}+\left(y_{n}-y_{n-1}\right)^{2}}
$$

The distance above the maximum distance between each node; a threshold distance $d_{T H}$ will be set to $5 \mathrm{~m}$. Based on the threshold distance $d_{T H}$, the check interval of the channel is changed depending on the if dist $\geq d_{T H}$. This will enable the nodes to know the node that is moving and the node that is static so as to decide which nodes to communicate with. The pseudocode of the proposed model is illustrated thus:

Pseudocode of the Mobility Conscious Scheme

Step 0: Let the nodes position be represented by $x$ and $y$. Let distance between nodes be represented by dist. Let the threshold distance be represented by $d_{T H}$. Let the floor estimation be represented by $f$. 
Step 1: Generate mobility patterns using $x$ and $y$.

Step 2: Get nodes distance from each other using dist.

Step 3: Set $d_{T H}$

Step 4: change check interval if dist $\geq d_{T H}$

Step 5: Add the information to the neighbourhood list of nodes.

Step 6: Set initial back off

Step 7: Check the channel to determine if it is clear using noise floor estimation.

Step 8: If channel is busy receive data or transmit data else sleep

Step 9: Stop

\section{Conclusion}

In this paper, attempt to model mobility pattern using Gauss Markov Mobility Model was achieved. The mathematical model was formulated with a view to determine the position and distance of the sensor nodes and how they are correlated in time. This model used the information of the distance between sensor nodes to check interval of the channel in order to provide ease of communication. This has a substantial effect on reducing the energy consumption and improving the throughput between sensor nodes. Differently from all other relevant heuristics approaches, our approach enhances faster connection and more time for sensor nodes to sleep.

\section{Further Works}

Future works will be to evaluate the mobility scenario for the sensor nodes. This will be done using an appropriate simulation tool and the performance metrics of interest. The results of the simulation will be benchmarked with the existing schemes in order to determine its efficiency.

\section{References}

[1] Ali, M., Suleman, T. and Uzmi, Z.A. (2005) MMAC: A Mobility-Adaptive, Collision-Free MAC Protocol for Wireless Sensor Networks. Proceedings of the 24 ${ }^{\text {th }}$ IEEE IPCCC'05, California, 401-407.

[2] Akyildiz, I.F., Su, W., Sankarasubramaniam, Y. and Cayirci, E. (2002) Wireless Sensor Networks: A Survey. Elsevier Journals Computer Networks, 38, 393-422. http://dx.doi.org/10.1016/S1389-1286(01)00302-4

[3] Pham, H. and Jha, S. (2004) An Adaptive Mobility-Aware MAC Protocol for Sensor Networks (MS-MAC). Proceedings of the IEEE International Conference on Mobile Ad-hoc and Sensor Systems (MASS-2004), 58-560. http://dx.doi.org/10.1109/MAHSS.2004.1392207

[4] Zhao, J., Sun, X., Wei, Z. and Li, Z. (2011) A New MAC Protocol for Moving Target in Distributed Wireless Sensor Networks. Journal of Wireless Sensor Network, 3, 61-72. http://www.scrip.org/journal/wsn

[5] Ye, W., Heidemann, J. and Estrin, D. (2002) An Energy-Efficient MAC Protocol for Wireless Sensor Networks. IEEE INFOCOM, 1567-1576.

[6] Dam, T.V. and Langendoen, K. (2003) An Adaptive Energy-Efficient MAC Protocol for Wireless Sensor Networks. The First ACM Conference on Embedded Networked Sensor Systems (Sensys'03), Los Angeles, 171-180.

[7] Rajendran, V., Obraczka, K. and Garcia-Luna-Aceves, J.J. (2003) Energy Efficient Collision Free Medium Access Control for Wireless Sensor Networks. Proceedings of ACM SenSys 03, 181-192.

[8] El-Hoiydi, A., Decotignie, J.D. and Hernandez, J. (2004) Low Power MAC Protocol for Infrastructure Wireless Sensor Networks. Proceedings of European Wireless (EW04), Barcelona, 563-569.

[9] Polastre, J. Hill, J. and Culler, D. (2004) Versatile Low Power Media Access for Wireless Sensor Networks. Proceedings of the Second ACM Conference on Embedded Networked Sensor System, Baltimore, 95-107.

[10] Van, H. and Havinga, P.J. (2004) A Lightweight Medium Access Protocol (LMAC) For Wireless Sensor Networks: 1st International Workshop on Networked Sensing Systems (INSS), Tokyo, 205-208.

[11] Suh, C. and Ko, Y.B. (2005) A Traffic Aware, Energy Efficient MAC Protocol for Wireless Sensor Networks. Proceeding of IEEE International Symposium on Circuit and Systems (IEEE/ISCS), 2975-2978.

[12] Zheng, T. and Sarangan, V. (2005) PMAC: An Adaptive Energy-Efficient MAC Protocol for Wireless Sensor Networks. Proceedings of 19th IEEE International Parallel and Distributed Processing Symposium, 237-244. http://dx.doi.org/10.1109/IPDPS.2005.344

[13] Mank, S., Karnapke, R. and Nolte, J. (2007) An Adaptive TDMA based MAC Protocol for Mobile Wireless Sensor 
Networks: IEEE Magazine in International Conference on Sensor Technologies and Applications (SensorComm), 6269.

[14] Miladi, M., Ezzedine, T. and Bouallegue, R. (2006) Latency of Energy Efficient MAC Protocols for Wireless Sensor Networks. IEEE Magazine in International Conference on Digital Telecommunications (ICDT), 29-31.

[15] Raja, A. and Su, X. (2009) Mobility Handling in MAC for Wireless Ad Hoc Networks. Wireless Communication and Mobile Computing, 9, 303-311. http://dx.doi.org/10.1002/wcm.613

[16] Titiloye, T.D. (2012) Development of an Improved Mobility Conscious Medium Access Control Scheme for Wireless Sensor Networks. Unpublished M.Sc. Thesis in the Department of Computer Science and Engineering Ile-Ife, Nigeria.

[17] Liang, B. and Haas, Z. (1999) Predictive Distance-based Mobility Management for PCS Networks. Proceedings of the IEEE international Conference on Computer Communications, 1377-1384.

[18] Tolety, V. (1999) Load Reduction in Ad Hoc Networks Using Mobile Servers. Published Master's Thesis, Colorado School of Mines, USA.

[19] Camp, T., Boleng, J. and Davies, V. (2002) A Survey of Mobility Models for Ad Hoc Network Research. Wireless Communication and Mobile Computing (WCMC): Special Issue on Mobile Ad Hoc Networking: Research, Trends and Applications, 2, 483-502. 
Scientific Research Publishing (SCIRP) is one of the largest Open Access journal publishers. It is currently publishing more than 200 open access, online, peer-reviewed journals covering a wide range of academic disciplines. SCIRP serves the worldwide academic communities and contributes to the progress and application of science with its publication.

Other selected journals from SCIRP are listed as below. Submit your manuscript to us via either submit@scirp.org or Online Submission Portal.
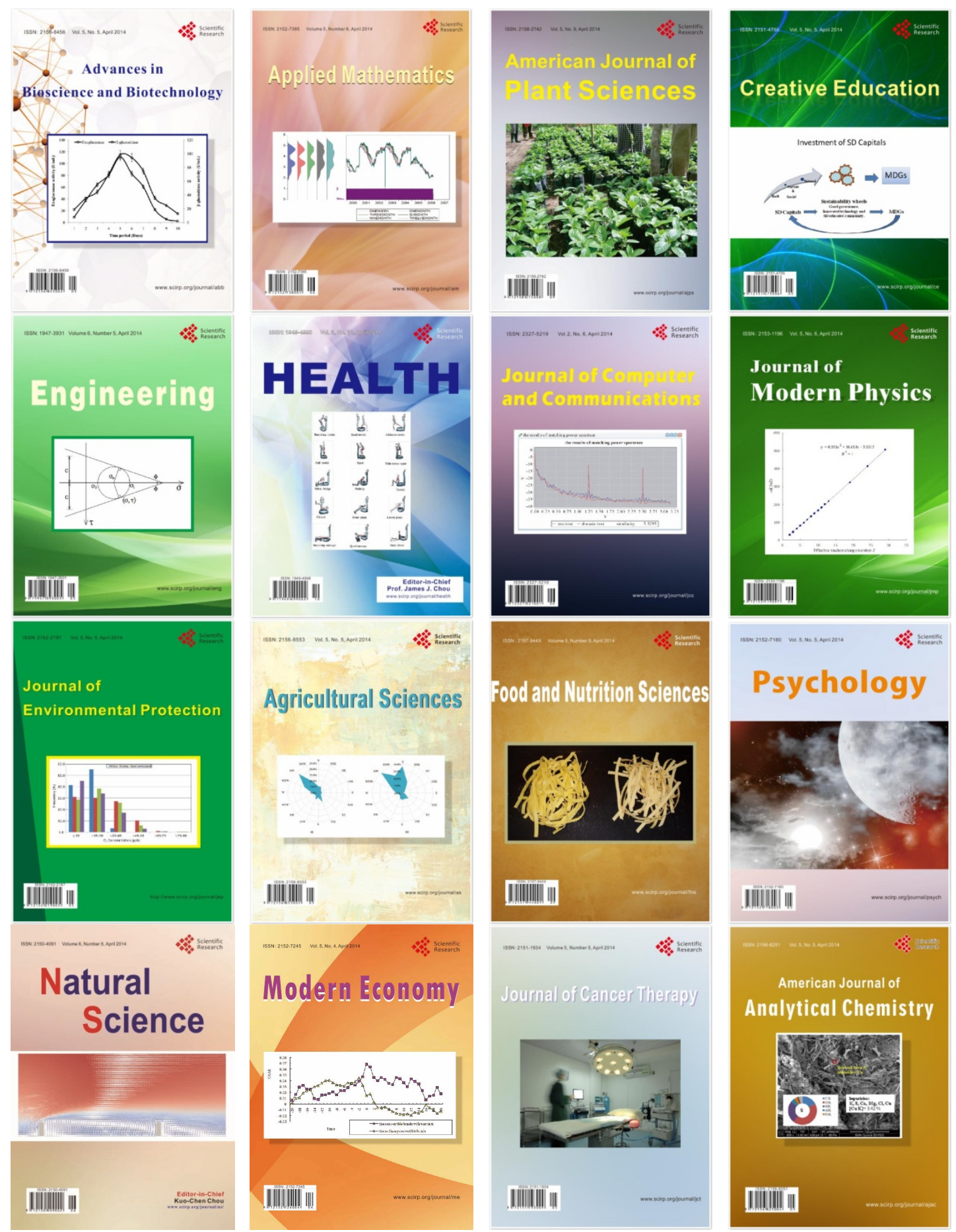